\title{
Perceived exertion and heart rate models for estimating metabolic workload in elite British soldiers performing a backpack load-carriage task
}

\author{
Richard J. Simpson, Scott M. Graham, Geraint D. Florida-James, \\ Christopher Connaboy, Richard Clement, and Andrew S. Jackson
}

\begin{abstract}
Identifying field measures to estimate backpack load-carriage work intensity in elite soldiers is of interest to the military. This study developed rating of perceived exertion (RPE) and heart rate models to define metabolic workload for a backpack load-carriage task valid for a population of elite soldiers using serial data. Male soldiers $(n=18)$ from the British Parachute or Special Air Service Regiment completed an incremental treadmill walking and (or) running protocol while carrying a $20 \mathrm{~kg}$ backpack. Heart rate, RPE, and oxygen uptake were recorded at each incremental stage of the protocol. Linear mixed models were used to model the RPE and heart rate data in the metric of measured peak oxygen uptake. Workload was accurately estimated using RPE alone ( $\mathrm{SE}=6.03)$, percentage of estimated maximum heart rate $(\% \mathrm{E}-\mathrm{MHR})(\mathrm{SE}=6.9)$, and percentage of measured maximum heart rate $(\% \mathrm{M}-\mathrm{MHR})(\mathrm{SE}=4.9)$. Combining RPE and $\%$ E-MHR resulted in a field measure with an accuracy $(\mathrm{SE}=4.9)$ equivalent to the \% M-MHR model. We conclude that RPE, \%E-MHR, and \%M-MHR provide accurate field-based proxy measures of metabolic workload in elite British soldiers performing a backpack load-carriage task. The model is accurate for the metabolic range measured by these serial data for the backpack load-carriage task.
\end{abstract}

Key words: linear mixed models, military, field test, oxygen uptake, RPE.

Résumé : Le milieu militaire s'intéresse particulièrement aux mesures sur le terrain pour estimer l'intensité de travail suscitée par le port d'un sac à dos chez des soldats d'élite. Cette étude présente à partir de données sérielles des modèles basés sur la perception de l'intensité de l'effort (RPE) et sur la fréquence cardiaque pour estimer l'intensité métabolique suscitée par le port d'un sac à dos chez des soldats d'élite. Dix-huit soldats masculins appartenant au Régiment britannique des parachutistes ou du SAS participent à une épreuve d'effort progressif fait de marche et de course tout en portant au dos une charge de $20 \mathrm{~kg}$. On enregistre les mesures de la fréquence cardiaque, de la RPE et de la consommation d'oxygène à chaque palier de l'épreuve d'effort. On utilise des modèles linéaires mixtes pour estimer la consommation d'oxygène de pointe au moyen de la RPE et de la fréquence cardiaque. On obtient une estimation précise de l'intensité de travail à partir de la RPE seule $(\mathrm{ET}=6,03)$, du pourcentage de la fréquence cardiaque maximale estimée $(\% \mathrm{E}-\mathrm{MHR})$ $(\mathrm{ET}=6,9)$ et du pourcentage de la fréquence cardiaque maximale mesurée $(\% \mathrm{M}-\mathrm{MHR})(\mathrm{ET}=4,9)$. En combinant la RPE et le $\% \mathrm{E}-\mathrm{MHR}$, la précision $(\mathrm{ET}=4,9)$ de la mesure sur le terrain est équivalente au modèle incluant le \%M-MHR. En conclusion, la RPE, le \%E-MHR et le \%M-MHR sont des variables substitutives donnant des mesures précises sur le terrain de l'intensité métabolique observée chez des soldats britanniques réalisant une tâche avec un sac au dos. Le modèle convient pour l'étendue des mesures du métabolisme obtenues lors de la prise des données sérielles s'appliquant au port du sac à dos.

Mots-clés : modèles linéaires mixtes, militaire, test sur le terrain, consommation d'oxygène, RPE.

[Traduit par la Rédaction]

\section{Introduction}

The elite units of the British armed forces require soldiers to march for prolonged periods of time carrying backpack loads that often amount to a high percentage (i.e., 25\%$50 \%$ ) of their body mass (Allsopp et al. 2004; Simpson et

\footnotetext{
Received 6 December 2009. Accepted 10 June 2010. Published on the NRC Research Press Web site at apnm.nrc.ca on .

R.J. Simpson. ${ }^{1}$ Laboratory of Integrated Physiology, Department of Health and Human Performance, University of Houston, 3855 Holman Street, Houston, TX 77204, USA; Biomedicine and Sports Science Research Group, School of Life Sciences, Edinburgh Napier University, 10 Colinton Road, Edinburgh, Scotland, EH10 5DT, UK.

S.M. Graham. School of Science and Technology, University of the West of Scotland, Paisley, Scotland, PA1 2AB, UK.

G.D. Florida-James, C. Connaboy, and R. Clement. Biomedicine and Sports Science Research Group, School of Life Sciences, Edinburgh Napier University, 10 Colinton Road, Edinburgh, Scotland, EH10 5DT, UK.

A.S. Jackson. Laboratory of Integrated Physiology, Department of Health and Human Performance, University of Houston, 3855 Holman Street, Houston, TX 77204, USA.

${ }^{1}$ Corresponding author (e-mail: rjsimpson@uh.edu).
} 
al. 2006; Wilkinson et al. 2008). Soldiers typically march in groups at defined speeds (Rayson et al. 2000; Allsopp and Shariff 2004; Christie et al. 2005; Simpson et al. 2006; Beekley et al. 2007; Wilkinson et al. 2008), meaning that the relative intensity of the workload will vary among soldiers, depending on their maximal oxygen uptake $\left(\dot{V} \mathrm{O}_{2} \max \right)$. Identifying field measures to estimate backpack load-carriage work intensity in soldiers is an important military concern (Rayson et al. 2000; Allsopp and Shariff 2004; Knapik et al. 2004; Simpson et al. 2006). Although the relative workload of a soldier (i.e., the percentage of $\dot{V} \mathrm{O}_{2 \max }$ ) is an important determinant of performance (Rayson et al. 2000; Bilzon et al. 2001; Christie and Scott 2005; Lyons et al. 2005), there are currently no field methods to accurately estimate workload during backpack load-carriage tasks in elite soldiers.

Many previous attempts have been made to estimate metabolic workload and $\dot{V} \mathrm{O}_{2}$ max using surrogate measures (Chen et al. 2002; Eston et al. 2006; Faulkner and Eston 2007 Faulkner et al 2007; Lambrick et al. 2009). The most common field measures that have been used to estimate exercise intensity (defined by $\%$ of $\dot{V} \mathrm{O}_{2}$ max ) are heart rate and perceived exertion. Borg's 6-20 rating of perceived exertion (RPE) scale (Borg 1998) is a surrogate measure used to estimate exercise intensity in a field setting. Numerous studies have been published relating RPE ratings to different criteria of exercise exertion, including running speed, oxygen uptake, ventilation, and heart rate. Chen et al. (2002) published a meta-analysis that summarized the RPE validity coefficients with 6 physiological criteria. The reported 95\% confidence interval of the meta validation coefficients between Borg's 6-20 RPE and each criterion were as follows: heart rate, 0.55 to 0.73 ; blood lactate, 0.67 to $0.77 ; \% \dot{V} \mathrm{O}_{2} \max$, 0.62 to $0.73 ; \dot{V} \mathrm{O}_{2}, 0.74$ to 0.80 ; ventilation, 0.70 to 0.79 ; and respiration rate, 0.60 to 0.80 . Although these data document the fact that RPE is related to physiological exertion, a limitation of this research is that these correlations were estimated using serial data within individual subjects. This generates bias estimates, because linear regression models do not take into account the correlations among the individual serial data (within-subject measures), increasing the probability of bias and inaccurate estimates of physiological exercise intensity. Linear mixed models (LMM) regression (Rabe-Hesketh 2008), a relatively new statistical method, allows a more valid means of modeling data, consisting of between- and within-subject measures. An additional advantage of LMM is that the method handles data when subjects do not have an equal number of observations.

The RPE validity coefficients reported by Chen et al. (2002) suggest that RPE can be used as a noninvasive measure to monitor work intensity in the field, but researchers have suggested that RPE may not accurately estimate exercise intensity and that its validity varies by the type of activity performed (e.g., running, cycling, swimming), the intensity of exercise, and the criterion physiological measure (Smutok et al. 1980; Eston et al. 1988; Whaley et al. 1997; Lamb et al. 1999; Chen et al. 2002; Garcin et al. 2003a; Novas et al. 2003). Furthermore, it is not known if RPE will be an accurate measure of exercise intensity during backpack load-carriage tasks in elite soldiers. The purpose of this study was to develop RPE and heart rate models to define exercise intensity for a backpack load-carriage task valid for a population of elite soldiers using serial data. The accuracy of the developed model was compared with physiological heart rate exercise intensity models. The goal was to develop an accurate field model with serial data that can be used by the military to monitor the relative workload of soldiers in field settings.

\section{Materials and methods}

\section{Participants}

Eighteen male soldiers of the British Army volunteered to participate in this study. All participants were serving with the Parachute Regiment or the Special Air Service Regiment and had passed preparachute selection and (or) United Kingdom Special Forces selection prior to participation in this study. A wide range of physiological variables and performance markers between soldiers in these 2 regiments were previously compared, and no discernible differences were found (Simpson et al. 2006). All participants had completed and passed their unit-specific annual physical fitness tests within the 12 months preceding the study, and refrained from strenuous physical activity for $48 \mathrm{~h}$ before participating in the exercise trials. All participants provided written informed consent, and ethical approval was granted by a local committee for human subjects research at Edinburgh Napier University (Edinburgh, Scotland). The physical characteristics of the participants are presented in Table 1.

\section{Incremental backpack treadmill protocol}

On arrival at the laboratory, each soldier was fitted with a heart rate monitor (S610, Polar Electro, Kempele, Finland) and asked to rest in the supine position on a plinth for $10 \mathrm{~min}$. After the 10-min rest, heart rate was recorded, and participants provided their RPE, using the Borg 6-20 scale (Borg 1998). All soldiers were instructed to consider their overall perception of the effort when providing their RPE. Each participant was fitted with a face mask for gas analysis purposes before putting on a standard British Army Bergan (backpack), which weighed $20 \mathrm{~kg}$ and was packed according to the comfort of the participant. The protocol began with the subject walking on a $+1 \%$ incline at a velocity of $6.4 \mathrm{~km} \cdot \mathrm{h}^{-1}$ on a motorized treadmill (Woodway Ergo, ELG 55, Weil am Rhein, Germany). The $1 \%$ incline was used to mimic the energy cost of outdoor running on a treadmill (Jones and Doust 1996). The velocity of the treadmill was then increased by $1.0 \mathrm{~km} \cdot \mathrm{h}^{-1}$ every 3 min until a maximal running velocity of $12.4 \mathrm{~km} \cdot \mathrm{h}^{-1}$ was attained. After 3 min of running at the maximal velocity, the inclination of the treadmill was increased by $2.5 \%$ every minute until volitional exhaustion. Heart rate and oxygen uptake were recorded throughout the test, and RPE was collected at the final $30 \mathrm{~s}$ of each incremental stage and on completion of the protocol. Oxygen uptake was measured continuously, using online gas analysis (CPX Medgraphics, Oldham, UK), and the mean oxygen uptake for every 10 breaths was recorded. Heart rate data were downloaded using the Polar interface, and the mean heart rate for each $5 \mathrm{~s}$ of the protocol was recorded. Maximum heart rate and $\dot{V} \mathrm{O}_{2}$ peak were determined from the test. $\dot{V} \mathrm{O}_{2}$ peak was identified as the highest oxygen uptake obtained over a 10-breath average. 
Table 1. Physical characteristics of the participants $(n=18)$.

\begin{tabular}{llll}
\hline Physical characteristics & Mean & SD & Range \\
\hline Age $(\mathrm{y})$ & 26.8 & 5.4 & $21.0-35.0$ \\
Height $(\mathrm{cm})$ & 179.8 & 6.3 & $168.5-189.5$ \\
Mass $(\mathrm{kg})$ & 79.9 & 6.9 & $67.0-87.4$ \\
Body mass index $\left(\mathrm{kg} \cdot \mathrm{m}^{-2}\right)$ & 24.8 & 2.8 & $20.0-29.0$ \\
$\mathrm{Backpack}$ load $(\%$ body mass) & 25.2 & 2.3 & $22.9-29.9$ \\
$\dot{V} \mathrm{O}_{2}$ peak $\left(\mathrm{L} \cdot \mathrm{min}^{-1}\right)$ & 4.33 & 0.37 & $3.60-5.00$ \\
$\dot{V} \mathrm{O}_{2}$ peak $\left(\mathrm{mL} \cdot \mathrm{kg}^{-1} \cdot \mathrm{min}^{-1}\right)$ & 54.5 & 5.4 & $46.0-62.3$ \\
$\mathrm{HR}_{\text {max }}\left(\right.$ beats$\left.\cdot \mathrm{min}^{-1}\right)$ & 196 & 10 & $179-213$ \\
Age-predicted $\mathrm{HR}_{\max }\left(\right.$ beats$\left.\cdot \mathrm{min}^{-1}\right)$ & 193 & 5 & $185-199$ \\
Maximal treadmill time (min:s) & $23: 18$ & $1: 13$ & $21: 00-24: 24$
\end{tabular}

Note: $\mathrm{HR}$, heart rate; $\dot{V} \mathrm{O}_{2 \text { peak, }}$, peak oxygen uptake.

\section{Statistical methods}

Maximum likelihood, LMM regression was used to model the data. A random intercept model was used for the LMM data analysis (Rabe-Hesketh 2008). Stata version 11 (xtmixed program) was used for data analysis (available from http://www.stata.com). The dependent variable for all models was $\% \dot{V} \mathrm{O}_{2}$ peak. The independent variable was Borg's 6-20 RPE rating with linear and quadratic terms. The accuracy of the RPE model was compared with percentage of maximum heart rate models, which were used 2 ways. First, age was used to estimate maximum heart rate (\%E-MHR), using the following equation: 220 - age. The second method used measured maximum heart rate (\%M-MHR). A log-ratio test was used to evaluate the fit between various models (Rabe-Hesketh 2008). Each fixedeffect LMM regression coefficient was tested with a $z$ test to determine if it was significantly different from 0 (RabeHesketh 2008). The random part of the LMM was the serial RPE and heart rate data produced from the multiple treadmill velocities. The accuracy of each model was estimated with the SE of the estimate. The $95 \%$ confidence intervals (CI) of the SE were computed to estimate equation accuracy of the population of elite soldiers carrying a backpack.

\section{Results}

Table 1 provides the physical characteristics and maximum exercise parameters of the participants. Table 2 gives the means and standard deviations for the exercise heart rate, RPE, and exercise intensity variables, contrasted by treadmill velocity. The 18 subjects completed all 7 treadmill speeds; however, the data for 1 subject were dropped because of erroneous metabolic data at the $6.4 \mathrm{~km} \cdot \mathrm{h}^{-1}$ velocity. The descriptive statistics show the well-documented positive association between changes in RPE and exercise heart rate with increased exercise intensity. The level of exercise intensity of the 18 soldiers at the $12.4 \mathrm{~km} \cdot \mathrm{h}^{-1}$ intensity ranged from $76.2 \%$ to $96.3 \% \dot{V} \mathrm{O}_{2}$ peak. The RPE at this intensity ranged from 15 to 18 .

Figure 1 depicts the relationship between $\% \dot{V} \mathrm{O}_{2}$ peak and RPE. The trend of the bivariate distribution suggested that the relation between $\% \dot{V} \mathrm{O}_{2}$ peak and RPE was not linear. An RPE quadratic term was added to the model, and the log-ratio test confirmed that the quadratic model provided a better fit of the data than the linear model $\left(\chi^{2}=8.26 ; p\right.$ $=0.004)$. Both the linear and quadratic modeled regression lines are shown in Fig. 1. The linear and quadratic regression equations for RPE and $\% \dot{V} \mathrm{O}_{2}$ peak are provided in Table 3. Figure 1 shows that the linear and quadratic models are nearly identical between an RPE of about 9 $\left(\approx 53 \% \dot{V} \mathrm{O}_{2 \text { peak }}\right)$ and $16\left(\approx 84 \% \dot{V} \mathrm{O}_{2 \text { peak }}\right)$. Above an RPE of 16, the quadratic estimate was lower than the linear estimate. Table 4 provides the quadratic $\% \dot{V} \mathrm{O}_{2}$ peak estimates for RPE ratings from 6 to 20. The SE of the quadratic equation for the fixed part of the model was $6.03 \% \dot{V} \mathrm{O}_{2}$ peak $(95 \% \mathrm{CI}=4.69$ to 7.96$)$.

Figure 2 gives the bivariate plot of residuals for the quadratic RPE models by RPE. The residuals are the difference between measured $\% \dot{V} \mathrm{O}_{2}$ peak and that estimated with the quadratic LMM equation. Provided are the residuals for the fixed and the random parts of the model. The random models include the fixed equation, and also account for the independent, reliable variance unique within each soldier. The $\mathrm{SE}$ of the random model was $4.57 \% \dot{\mathrm{VO}} \mathrm{O}_{2}$ peak $(95 \% \mathrm{CI}=$ 3.45 to 4.51 ), and represents the lowest $S E$ theoretically possible for the RPEdata. The plot of the residuals shows that the largesterrors are for RPE ratings between $11\left(\approx 63 \% \dot{V} \mathrm{O}_{2 \text { peak }}\right)$ and 15 ( $\approx 80 \% \dot{V} \mathrm{O}_{2}$ peak). For reference, in Fig. 2, lines for residuals of $0 \%$ and $\pm 10 \%$ are shown. There were 12 fixed-effect estimates (or $9.6 \%$ of all observations) with errors beyond $\pm 10 \%$, and 3 (or $2.4 \%$ of all observations) random estimates beyond $\pm 10 \%$. The residuals for both the fixed and random models were examined graphically in 2 ways: normal curves superimposed on the histogram of residuals; and standardized normal quartile probability plots of the residuals (Rabe-Hesketh 2008). These graphs showed that the residuals were normally distributed (graphics not shown), with no serious outliers.

Table 5 provides the LMM with functions to estimate exercise intensity with the percentage of maximum heart rate variables. The heart rate models were developed to provide a comparison with the RPE accuracy. The SE of the $\%$ E-MHR model was 6.90 (95\% CI $=5.21$ to 9.40), which was slightly higher than the RPE model. The \%M-MHR model was the most accurate model, with a fixed-effect $\mathrm{SE}<5 \%$. As another comparison, RPE was added to the heart rate models. Log-ratio tests confirmed that adding RPE to the heart rate models improved the fit $(p<0.001)$ for both heart rate terms. This showed that the RPE and the heart rate models accounted for independent sources of exercise intensity variance, and the combined RPE and percent heart rate models provided the most accurate estimates of exercise intensity.

\section{Discussion}

In this study, we developed RPE and heart rate models using serial data to estimate metabolic workload during a backpack load-carriage task in elite British soldiers. The novel features of this study include the use of LMM to develop the prediction models with serial data, and the application of RPE and heart rate as surrogate measures of metabolic workload to a population of elite British soldiers performing a backpack load-carriage task. We found that workload could be estimated with just RPE with a SE of $6.03 \% \dot{V} \mathrm{O}_{2}$ peak. For the percentage of estimated maximum 
Table 2. Descriptive statistics of exercise data, contrasted by treadmill speed.

\begin{tabular}{|c|c|c|c|c|c|c|}
\hline \multirow[b]{2}{*}{ Treadmill speed $\left(\mathrm{km} \cdot \mathrm{h}^{-1}\right)^{*}$} & \multicolumn{2}{|c|}{$\begin{array}{l}\text { Exercise heart rate } \\
\text { (beats } \cdot \mathrm{min}^{-1} \text { ) }\end{array}$} & \multicolumn{2}{|c|}{$\begin{array}{l}\text { Borg's 6-20 RPE } \\
\text { (rating) }\end{array}$} & \multicolumn{2}{|c|}{$\begin{array}{l}\text { Exercise intensity } \\
\left(\% \dot{V} \mathrm{O}_{2 \max }\right)\end{array}$} \\
\hline & Mean & SD & Mean & SD & Mean & SD \\
\hline 6.4 & 112.5 & 11.0 & 7.4 & 0.7 & 43.5 & 3.9 \\
\hline 7.4 & 132.0 & 12.1 & 9.9 & 0.9 & 56.2 & 4.1 \\
\hline 8.4 & 148.2 & 14.3 & 11.8 & 0.9 & 68.5 & 4.6 \\
\hline 9.4 & 155.4 & 14.0 & 12.9 & 0.8 & 70.9 & 5.6 \\
\hline 10.4 & 161.4 & 14.2 & 13.7 & 0.9 & 75.4 & 5.7 \\
\hline 11.4 & 168.8 & 13.9 & 15.0 & 1.0 & 80.8 & 6.2 \\
\hline 12.4 & 175.4 & 12.9 & 16.6 & 0.9 & 86.1 & 5.3 \\
\hline
\end{tabular}

Note: RPE, rating of perceived exertion; $\% \dot{V} \mathrm{O}_{2 \max }$, percentage of maximal oxygen uptake.

*Sample size is 17 for $6.4 \mathrm{~km} \cdot \mathrm{h}^{-1}$ and 18 for the other speeds.

heart rate model, the SE was slightly less accurate, at $6.9 \% \dot{V} \mathrm{O}_{2}$ peak, while the measured maximum heart rate model was the most accurate $\left(\mathrm{SE}=4.9 \% \dot{V} \mathrm{O}_{2}\right.$ peak $)$. Combining $\mathrm{RPE}$ and \%E-MHR resulted in a field measure with accuracy $\left(\mathrm{SE}=4.9 \% \dot{\mathrm{VO}} \mathrm{O}_{2 \text { peak }}\right)$ equivalent to the model that used measured maximum heart rate. The model is accurate for the metabolic range measured by these serial data for the backpack load-carriage task.

While it is well documented that RPE is associated with exercise intensity (Chen et al. 2002), this is the first study, to our knowledge, to publish an unbiased model that calibrates Borg's 6-20 RPE in the metric of measured $\% \dot{V} \mathrm{O}_{2}$ peak using serial data. While others have estimated physiological parameters with RPE using linear regression (Smutok et al. 1980; Marriott and Lamb 1996; Garcin et al. 2003b; Novas et al. 2003; Mielke et al. 2008), the limitation of these studies is that serial data produce within-subject correlations among the residuals, resulting in bias error estimates (RabeHesketh 2008). An assumption of regression models is that the correlation between the residuals and estimated values is zero. The strength of LMM is that the random part of the model accounts for the within-subject variability, providing unbiased error estimates. Standard output of the Stata version 11 xtmixed program is a log-ratio test to determine if the LMM provide a more accurate fit of the data than simple regression. The log-ratio tests for all LMM provided in Tables 3 and 5 were significantly different $(p<0.001)$, supporting the need to use LMM with these serial data. The data in Fig. 2 demonstrate that the residuals and RPE are independent, further supporting the use of the LMM.

It is commonly assumed that Borg's 6-20 scale is linearly related to physiological intensity data. Heart rate, oxygen uptake, $\% \dot{V} \mathrm{O}_{2 \text { max }} / \% \dot{V} \mathrm{O}_{2 \text { peak }}$, blood lactate, ventilation, and respiratory rate have been the physiological criterion measures used in this research (Chen et al. 2002). A likely reason for this is that the 6-20 scale was developed to represent actual heart rate values from resting (i.e., $6 \times 10=60$ beats. $\mathrm{min}^{-1}$ ) to maximum (i.e., $20 \times 10=200$ beats $\cdot \mathrm{min}^{-1}$ ) (personal communication between G. Borg and A.S. Jackson, July 1994). These results show that the relationship between $\mathrm{RPE}$ and $\% \dot{V} \mathrm{O}_{2}$ peak is quadratic, not linear. The nonlinear relationship between exercise intensity and RPE is consistent with the theory used by Borg to develop his categoryratio (CR10) RPE scale. The CR10 scale is based on the assumption that, at higher intensity levels, a small increase in physiological intensity produces a larger increase in the perception of intensity (Borg 1998). This is perhaps because individuals are more aware of their exertions (e.g., ventilation, respiratory rate) at higher intensities of exercise than at lower intensities of exercise. The nonlinear relation between exercise intensity and RPE is consistent with Borg's theoretical position. Figure 2 shows that beyond an RPE of $16\left(83.7 \% \dot{V} \mathrm{O}_{2}\right.$ peak $)$, the curve changes at a progressively slower rate for each unit increase in RPE, and that $\% \dot{V} \mathrm{O}_{2}$ peak increases at a progressively slower rate. Indeed, it has been reported that RPE can be an inaccurate estimate of exercise intensity under certain conditions (Smutok et al. 1980; Eston and Williams 1988; Whaley et al. 1997; Lamb et al. 1999; Chen et al. 2002; Garcin et al. 2003a; Novas et al. 2003), which may be due to the nonlinear relationship between $\mathrm{RPE}$ and $\% \dot{\mathrm{V}} \mathrm{O}_{2}$ peak reported in our study.

An important finding of these analyses is that the SE for the RPE prediction model is slightly more accurate at predicting $\% \dot{V} \mathrm{O}_{2}$ peak than percent of maximum heart rate, when maximum heart rate is estimated by 220 - age. The limitation of using age to estimate maximum heart rate is clearly shown by the LMM analysis of the heart rate data. The SE for the \%E-MHR model of $6.90(95 \% \mathrm{CI}=5.21$ to $9.40)$ is much higher than for the \%M-MHR of $4.90(95 \%$ $\mathrm{CI}=3.79$ to 6.52 ). While exercise heart rate is relatively easy to measure using short-range telemetry, the advantage of RPE is that there is no need for specialist equipment in a field setting, which could be more suitable to the military when attempting to estimate workload in a number of soldiers during loaded marches.

Our results also show that if actual maximum heart rate (i.e., that measured objectively in response to a maximal exercise test) is used to estimate workload, then the heart rate model is more accurate than the RPE model as an estimate of exercise intensity. Although a limitation of the \% M-MHR model is that maximum heart rate must be measured, and heart rate monitors have to be worn by the soldiers, when working with elite units of the British Army, the additional max testing and the use of heart rate monitors can be balanced by the need for greater accuracy. Military units often monitor the progression of their recruits during basic training using maximal shuttle running tests (Pope et al. 1999; Demeritt et al. 2002; Rosendal et al. 2003). Clearly, measured maximum heart rate could be obtained from such tests, 
Fig. 1. Bivariate relationship between exercise intensity (percentage of peak oxygen uptake $\left(\% \dot{V} \mathrm{O}_{2}\right.$ peak $)$ ) and Borg's $6-20$ rating of perceived exertion scale in elite British soldiers performing an incremental backpack load-carriage task. The linear (dashed) and quadratic (solid) linear mixed model regression lines are provided for reference.

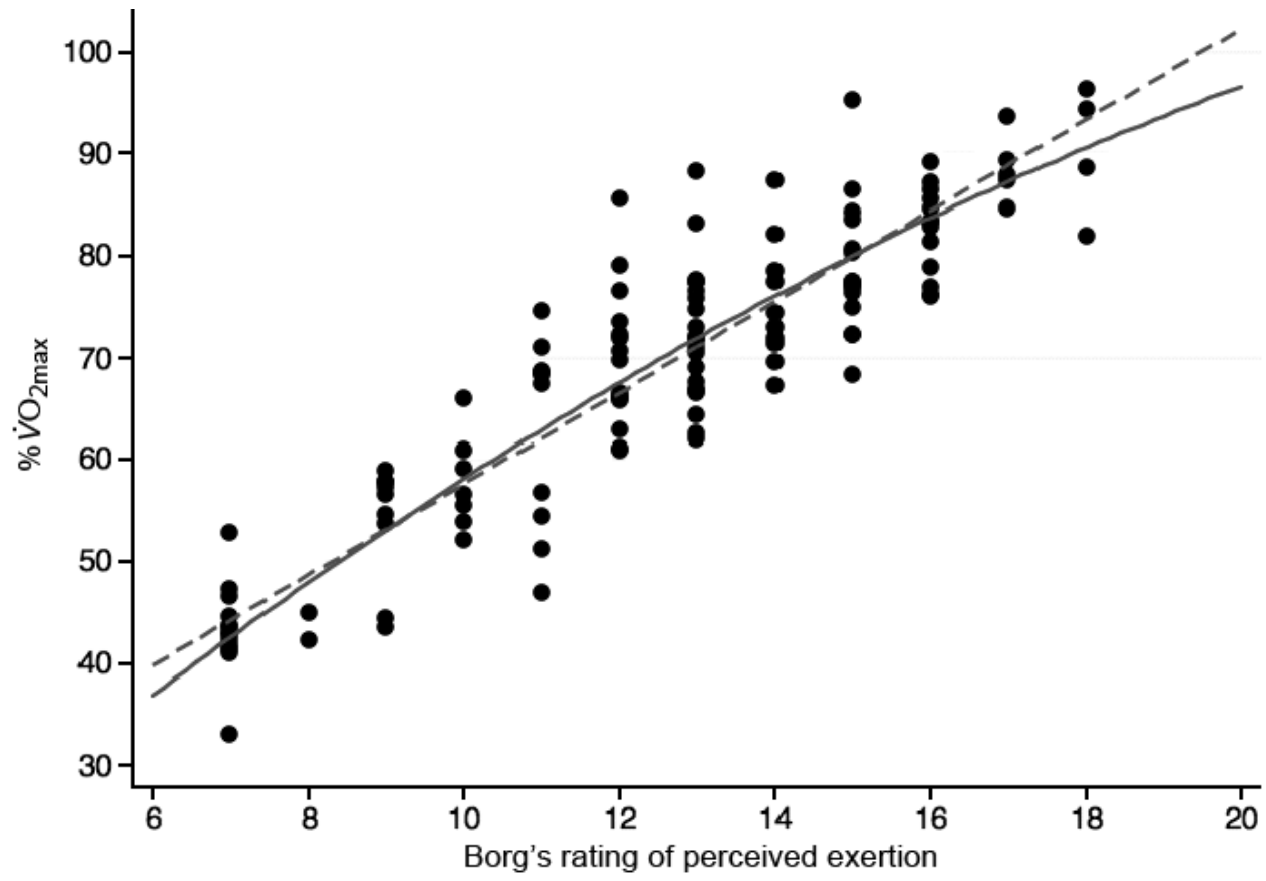

Table 3. Regression equations and SE estimates for estimating the percentage of peak oxygen uptake from Borg's 6-20 rating of perceived exertion scale for the fixed part of the linear mixed models.

\begin{tabular}{lll}
\hline Model & Equation & SE $(95 \% \text { CI })^{*}$ \\
\hline Linear & $\% \dot{V} \mathrm{O}_{2 \text { peak }}=(4.43 \times \mathrm{RPE})+13.54$ & $6.15(4.80-8.11)$ \\
Quadratic & $\% \dot{V} \mathrm{O}_{2 \text { peak }}=(7.08 \times \mathrm{RPE})-(0.11 \times \mathrm{RPE} 2)-1.38$ & $6.03(4.69-7.96)$ \\
\hline
\end{tabular}

Note: $\mathrm{CI}$, confidence interval; $\% \dot{V} \mathrm{O}_{2 \text { peak }}$, percentage of peak oxygen uptake; RPE, rating of perceived exertion.

*All regression coefficients significant, $p<0.01$.

Table 4. Calibration of Borg's 6-20 rating of perceived exertion scale in the metric of $\%$ peak oxygen uptake.

\begin{tabular}{ll}
\hline RPE & $\% \dot{V} \mathrm{O}_{2 \text { peak }}$ \\
\hline 6 & 36.9 \\
7 & 42.5 \\
8 & 47.9 \\
9 & 53.1 \\
10 & 58.1 \\
11 & 62.9 \\
12 & 67.5 \\
13 & 71.9 \\
14 & 76.0 \\
15 & 80.0 \\
16 & 83.7 \\
17 & 87.3 \\
18 & 90.6 \\
19 & 93.7 \\
20 & 96.6 \\
\hline
\end{tabular}

Note: RPE, rating of perceived exertion; $\% \dot{V} \mathrm{O}_{2 \text { peak }}$, percentage of peak oxygen uptake. and subsequently used in the field as a surrogate measure of workload during backpack load-carriage tasks. In addition, the equation that combines RPE with \%E-MHR provides a model with comparable accuracy to the \%M-MHR model and, hence, its use would also be appropriate.

Although it has been reported that the use of heart rate as a surrogate measure of oxygen uptake during a backpack load-carriage task is erroneous (Rayson et al. 1995), this only applies if the heart rate and oxygen uptake relationship is determined from an unloaded (i.e., without a backpack) running protocol. As our equations were generated from a protocol that established the heart rate and oxygen uptake relationship during an incremental backpack load-carriage task, this might explain why the percentage of maximum heart rate was an accurate proxy measure of $\% \dot{V} \mathrm{O}_{2}$ peak in our study. It has also been reported that the validity of the RPE varies, depending on the type of activity performed (Chen et al. 2002). To the best of our knowledge, we have used for the first time an unbiased model that calibrates Borg's 6-20 RPE in the metric of measured $\% \dot{V} \mathrm{O}_{2}$ peak using serial data, but it should be stressed that the equations generated in this study are limited to estimating exercise inten-

\section{PROOF/ÉPREUVE}


Fig. 2. Bivariate plot of the residuals for the quadratic rating of perceived exertion (RPE) model by RPE. Provided are the residuals for the fixed and random parts of the model. For reference, lines for residuals of $0 \%$ and $\pm 10 \%$ peak oxygen uptake $\left(\dot{V} \mathrm{O}_{2}\right.$ peak $)$ are shown.

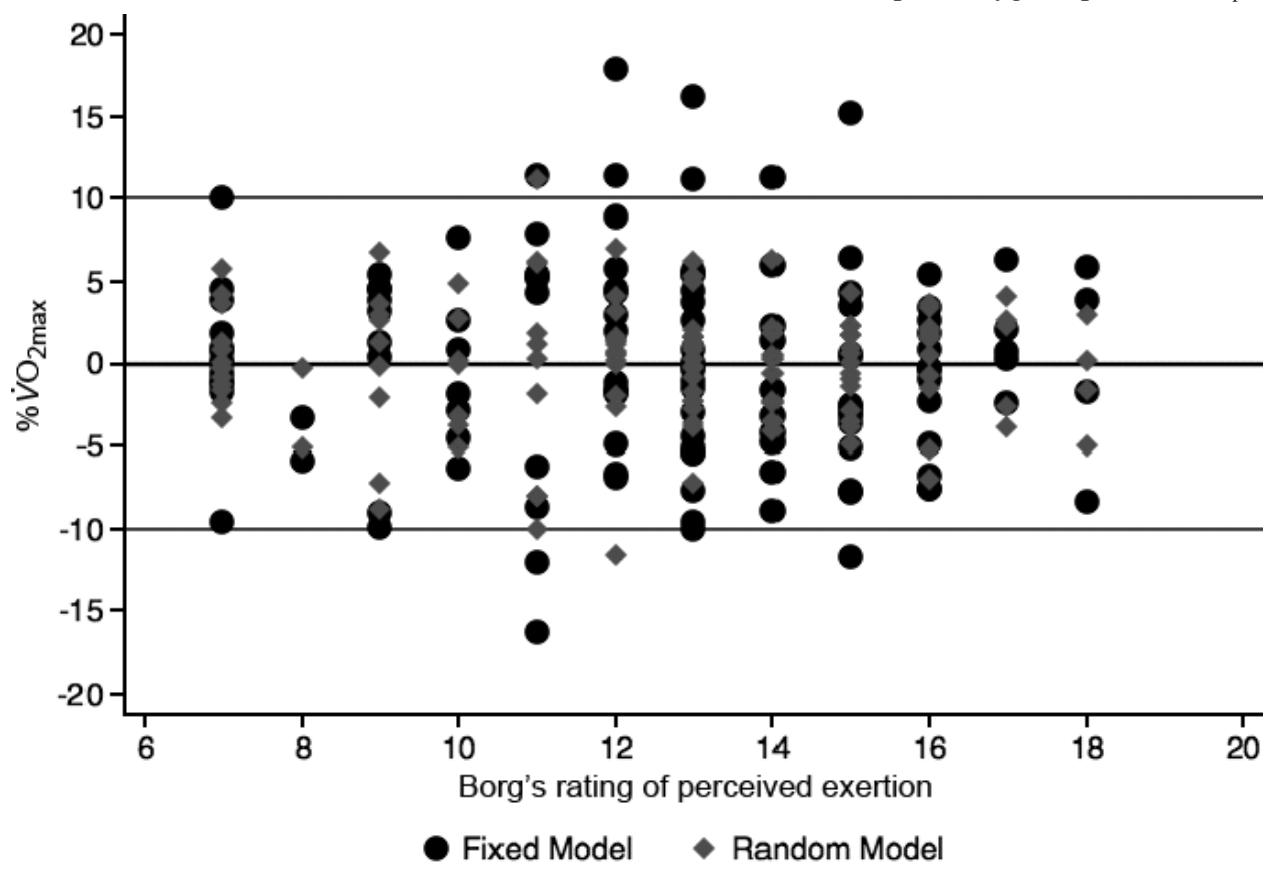

Table 5. Regression equations and SE estimates for estimating percentage of peak oxygen uptake from exercise heart rate and rating of perceived exertion for the fixed part of the linear mixed model.

\begin{tabular}{lll}
\hline Model & Equation & SE $(95 \%$ CI $)$ \\
\hline LMM-1 & $\% \dot{V} \mathrm{O}_{2 \text { peak }}=(1.23 \times \%$ E-MHR $)-26.64$ & $6.90(5.21-9.40)$ \\
LMM-2 & $\% \dot{V} \mathrm{O}_{2 \text { peak }}=(1.24 \times \%$ M-MHR $)-27.51$ & $4.90(3.79-6.52)$ \\
LMM-3 & $\% \dot{V} \mathrm{O}_{2 \text { peak }}=(0.73 \times \%$ E-MHR $)+(1.92 \times \mathrm{RPE})-12.10$ & $4.90(3.72-6.60)$ \\
LMM-4 & $\% \dot{V} \mathrm{O}_{2 \text { peak }}=(0.75 \times \% \mathrm{M}-\mathrm{MHR})+(1.89 \times \mathrm{RPE})-13.37$ & $3.52(2.80-4.60)$ \\
\hline
\end{tabular}

Note: $\mathrm{CI}$, confidence interval; $\% \dot{V} \mathrm{O}_{2 \text { peak }}$, percentage of peak oxygen uptake; RPE, rating of perceived exertion; LMM, linear mixed model; \%E-MHR, percentage of estimated maximum heart rate; $\%$ M-MHR, percentage of measured maximum heart rate.

sity during this backpack load-carriage task in elite British soldiers. Furthermore, these RPE and heart rate models may only be accurate at predicting workload in soldiers carrying an external backpack load of $20 \mathrm{~kg}$ at velocities ranging from 6.4 to $12.4 \mathrm{~km} \cdot \mathrm{h}^{-1}$. Other researchers would be advised to use different backpack loads and treadmill walking and running velocities to cross-validate these models. While our data can only validly be inferred to this particular task and subject cohort, this study does provide a benchmark for future cross-validation, calibration research. The use of a similar statistical approach to estimate exercise intensity in other tasks (e.g., running, cycling, rowing, walking) and among different subject cohorts (e.g., trained vs. untrained, male vs. female) will allow us to determine how generalized these models are at estimating workload using serial RPE and heart rate data.

These data demonstrate that RPE and percentage of maximum heart rate, using both estimated and measured maximum heart rate, provide field-based proxy measures of metabolic workload in elite British soldiers performing a backpack load-carriage task. The accuracy of the models are defined by the SE. The relation between $\% \dot{V} \mathrm{O}_{2} \max$ and
Borg's 6-20 RPE rating was nonlinear, consistent with the theory used to develop the CR10 RPE scale. The RPE model was slightly more accurate than the maximum heart rate model, when maximum heart rate was estimated from age. Combining RPE with \%E-MHR produced a model with accuracy equivalent to the heart rate model that measured maximum heart rate. These results demonstrate that LMM provide a better fit of these serial data than linear regression models.

\section{Acknowledgements}

This study was supported by the Carnegie Trust for the Universities of Scotland Student Vacation Scholarship for Richard Clement.

\section{References}

Allsopp, A.J., and Shariff, A. 2004. Improving the selection of candidates for Royal Marine recruit training by the use of a combination of performance tests. J. R. Nav. Med. Serv. 90(3): 117124. PMID: 15745257

Beekley, M.D., Alt, J., Buckley, C.M., Duffey, M., and Crowder, T.A. 2007. Effects of heavy load carriage during constant-speed, 
simulated, road marching. Mil. Med. 172(6): 592-595. PMID: 17615838 .

Bilzon, J.L., Allsopp, A.J., and Tipton, M.J. 2001. Assessment of physical fitness for occupations encompassing load-carriage tasks. Occup. Med. (Lond.), 51(5): 357-361. doi:10.1093/ occmed/51.5.357. PMID:11473145.

Borg, G. 1998. Borg's perceived exertion and pain scales. Human Kinetics, Champaign, Ill.

Chen, M.J., Fan, X., and Moe, S.T. 2002. Criterion-related validity of the Borg ratings of perceived exertion scale in healthy individuals: a meta-analysis. J. Sports Sci. 20(11): 873-899. doi:10. 1080/026404102320761787. PMID:12430990.

Christie, C.J., and Scott, P.A. 2005. Metabolic responses of South African soldiers during simulated marching with 16 combinations of speed and backpack load. Mil. Med. 170(7): 619-622. PMID:16130645.

Demeritt, K.M., Shultz, S.J., Docherty, C.L., Gansneder, B.M., and Perrin, D.H. 2002. Chronic ankle instability does not affect lower extremity functional performance. J. Athl. Train. 37(4): 507-511. PMID:12937575.

Eston, R.G., and Williams, J.G. 1988. Reliability of ratings of perceived effort regulation of exercise intensity. Br. J. Sports Med. 22(4): 153-155. doi:10.1136/bjsm.22.4.153. PMID:3228684.

Eston, R.G., Faulkner, J.A., Mason, E.A., and Parfitt, G. 2006. The validity of predicting maximal oxygen uptake from perceptually regulated graded exercise tests of different durations. Eur. J. Appl. Physiol. 97(5): 535-541. doi:10.1007/s00421-006-0213-x. PMID:16779551.

Faulkner, J., and Eston, R. 2007. Overall and peripheral ratings of perceived exertion during a graded exercise test to volitional exhaustion in individuals of high and low fitness. Eur. J. Appl. Physiol. 101(5): 613-620. doi:10.1007/s00421-007-0536-2. PMID:17694318.

Faulkner, J., Parfitt, G., and Eston, R. 2007. Prediction of maximal oxygen uptake from the ratings of perceived exertion and heart rate during a perceptually-regulated sub-maximal exercise test in active and sedentary participants. Eur. J. Appl. Physiol. 101(3): 397-407. doi:10.1007/s00421-007-0508-6. PMID:17684757.

Garcin, M., Mille-Hamard, L., Devillers, S., Delattre, E., Dufour, S., and Billat, V. 2003a. Influence of the type of training sport practised on psychological and physiological parameters during exhausting endurance exercises. Percept. Mot. Skills, 97(3 Pt. 2): 1150-1162. PMID:15002859.

Garcin, M., Wolff, M., and Bejma, T. 2003b. Reliability of rating scales of perceived exertion and heart rate during progressive and maximal constant load exercises till exhaustion in physical education students. Int. J. Sports Med. 24(4): 285-290. doi:10. 1055/s-2003-39502. PMID:12784171.

Jones, A.M., and Doust, J.H. 1996. A 1\% treadmill grade most accurately reflects the energetic cost of outdoor running. J. Sports Sci. 14(4): 321-327. doi:10.1080/02640419608727717. PMID: 8887211.

Knapik, J.J., Reynolds, K.L., and Harman, E. 2004. Soldier load carriage: historical, physiological, biomechanical, and medical aspects. Mil. Med. 169(1): 45-56. PMID:14964502.

Lamb, K.L., Eston, R.G., and Corns, D. 1999. Reliability of ratings of perceived exertion during progressive treadmill exercise. $\mathrm{Br}$.
J. Sports Med. 33(5): 336-339. doi:10.1136/bjsm.33.5.336. PMID: 10522637.

Lambrick, D.M., Faulkner, J.A., Rowlands, A.V., and Eston, R.G. 2009. Prediction of maximal oxygen uptake from submaximal ratings of perceived exertion and heart rate during a continuous exercise test: the efficacy of RPE 13. Eur. J. Appl. Physiol. 107(1): 1-9. doi:10.1007/s00421-009-1093-7. PMID:19488778.

Lyons, J., Allsopp, A., and Bilzon, J. 2005. Influences of body composition upon the relative metabolic and cardiovascular demands of load-carriage. Occup. Med. (Lond.), 55(5): 380-384. doi:10.1093/occmed/kqi087. PMID:15860484.

Marriott, H.E., and Lamb, K.L. 1996. The use of ratings of perceived exertion for regulating exercise levels in rowing ergometry. Eur. J. Appl. Physiol. Occup. Physiol. 72(3): 267-271. doi:10.1007/BF00838650. PMID:8820897.

Mielke, M., Housh, T.J., Malek, M.H., Beck, T.W., Schmidt, R.J., and Johnson, G.O. 2008. The development of rating of perceived exertion-based tests of physical working capacity. J. Strength Cond. Res. 22(1): 293-302. PMID:18296989.

Novas, A.M., Rowbottom, D.G., and Jenkins, D.G. 2003. A practical method of estimating energy expenditure during tennis play. J. Sci. Med. Sport, 6(1): 40-50. doi:10.1016/S1440-2440(03) 80007-5. PMID:12801209.

Pope, R.P., Herbert, R., Kirwan, J.D., and Graham, B.J. 1999. Predicting attrition in basic military training. Mil. Med. 164(10): 710-714. PMID:10544625.

Rabe-Hesketh, S.S.A. 2008. Multilevel and longitudinal modeling using stata. Stata Press, College Station, Tex.

Rayson, M.P., Davies, A., Bell, D.G., and Rhodes-James, E.S. 1995. Heart rate and oxygen uptake relationship: a comparison of loaded marching and running in women. Eur. J. Appl. Physiol. Occup. Physiol. 71(5): 405-408. doi:10.1007/BF00635873. PMID:8565971.

Rayson, M., Holliman, D., and Belyavin, A. 2000. Development of physical selection procedures for the British Army. Phase 2: relationship between physical performance tests and criterion tasks. Ergonomics, 43(1): 73-105. doi:10.1080/ 001401300184675. PMID:10661694.

Rosendal, L., Langberg, H., Skov-Jensen, A., and Kjaer, M. 2003. Incidence of injury and physical performance adaptations during military training. Clin. J. Sport Med. 13(3): 157-163. doi:10. 1097/00042752-200305000-00006. PMID:12792210.

Simpson, R.J., Gray, S.C., and Florida-James, G.D. 2006. Physiological variables and performance markers of serving soldiers from two "elite" units of the British Army. J. Sports Sci. 24(6): 597-604. doi:10.1080/02640410500230811. PMID:16611570.

Smutok, M.A., Skrinar, G.S., and Pandolf, K.B. 1980. Exercise intensity: subjective regulation by perceived exertion. Arch. Phys. Med. Rehabil. 61(12): 569-574. PMID:7458621.

Whaley, M.H., Woodall, T., Kaminsky, L.A., and Emmett, J.D. 1997. Reliability of perceived exertion during graded exercise testing in apparently healthy adults. J. Cardiopulm. Rehabil. 17(1): 37-42. doi:10.1097/00008483-199701000-00005. PMID: 9041069.

Wilkinson, D.M., Rayson, M.P., and Bilzon, J.L. 2008. A physical demands analysis of the 24-week British Army Parachute Regiment recruit training syllabus. Ergonomics, 51(5): 649-662. doi:10.1080/00140130701757367. PMID:18432443. 